\title{
Cooperative cation co-transport by supramolecular self-associating amphiphiles
}

Kylie Yang, ${ }^{a}, \dagger$ Jessica E. Boles, $,{ }^{b, c},{ }^{\dagger}$ Lisa J. White, ${ }^{b}$ Kira H. L. F. Hilton, ${ }^{b}$ Hin Yuk Lai, ${ }^{a}$ Yifan Long, $^{a}$ Jennifer R. Hiscock ${ }^{b *}$ and Cally J. E. Haynes ${ }^{a *}$

a Chemistry Department, UCL, 20 Gordon Street, London WC1H 0AJ, UK. Email: cally.haynes@ucl.ac.uk

b School of Physical Sciences, University of Kent, Canterbury, Kent, CT2 7NH, UK. E-mail: J.R.Hiscock@Kent.ac.uk

c School of Biosciences, University of Kent, Canterbury, Kent, CT2 7NJ, UK.

†These authors contributed equally.

\begin{abstract}
We report investigations into the cation transport ability of a series of antimicrobial supramolecular, self-associating amphiphiles (SSAs). The SSAs proved to be challenging to study using standard, literature assays and we therefore describe a novel methodology to examine cation transport via a chloride co-transport assay. One SSA in this series was observed to function as a $\mathrm{K}^{+}$and $\mathrm{Na}^{+}$transporter with promising deliverability properties. The results shed light on a potential mechanism of microbial toxicity and inform the design of future targets.
\end{abstract}

\section{Main article text}

Antimicrobial resistance (AMR) is a growing threat to humanity, with AMR now reported against all currently available antimicrobial agents. This has resulted in the need for the development of next generation antimicrobial therapeutic treatments, which includes the design and synthesis of novel antibiotics. ${ }^{1}$ Several novel approaches to the design of nextgeneration antibiotics have been inspired by our understanding of antimicrobial, pore forming peptides. ${ }^{2}$ These innovations include the development of ionophores that can selectively insert into and permeabilise Gram-positive (such as Staphylococcus aureus) and Gramnegative such as Escherichia coli) bacterial membranes enabling the movement of ions across cell membranes, triggering cell death. ${ }^{3-9}$ Recently reported examples of small molecule $\mathrm{K}^{+}$and $\mathrm{Na}^{+}$transporters have commonly utilised macrocycles such as crown ethers ${ }^{10-20}$ and strapped calix[4]pyrrole moieties. ${ }^{21}$ However, a limitation of many supramolecular ionophores designed for therapeutic applications is their high lipophilicity, making them poorly water soluble, thus limiting the translation of this molecular technology into the clinic. Improving the solubility and thereby bioavailability of lipophilic drugs remains one of the most challenging aspects of drug development, formulation delivery. ${ }^{22}$ In the case of lipophilic ionophores, researchers have investigated strategies to improve delivery to the therapeutic site of action including encapsulation within synthetic phospholipid vesicles, ${ }^{23}$ pro-drug strategies ${ }^{24}$ and complexation within water-soluble cyclodextrin macrocycles. ${ }^{25}$ These steps increase the likelihood of this class of molecules being adopted within the treatment of bacterial infections, cancer and channelopathies (disorders resulting from the dysfunction of ion channels). 4, 26, 27

Supramolecular, self-associating amphiphiles (SSAs) are a class of small molecule, amphiphilic salts developed by the Hiscock group that have been shown to act as antimicrobial agents ${ }^{28}$ and efficacy enhancers for both anticancer and antimicrobial agents. ${ }^{29-32}$ Recent studies have established that many SSAs, including the intrinsically fluorescent, benzothiazole substituted 1 and 2 (Figure 1), display aggregation-dependent antimicrobial activity against both Gram-positive MRSA USA300 and Gram-negative bacteria 
Escherichia coli (E. coli) DH10beta. ${ }^{28,} 33$ In particular, SSA 2 has been observed to coat and penetrate both bacterial cell and cancer cell membranes in fluorescence microscopy experiments. ${ }^{32,33}$ Given our knowledge of the antimicrobial mechanisms of pore-forming peptides and other ionophores, we now consider the questions: can SSAs promote membrane leakage or selective ion transport, and does this contribute to their established antimicrobial or antimicrobial/anticancer efficacy enhancement activity?

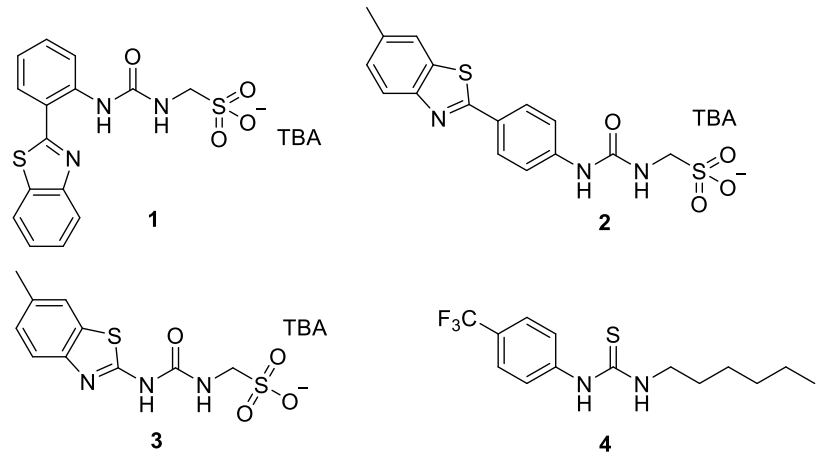

Figure $\mathbf{1}$ The structures of SSAs $\mathbf{1 - 3}$ and anionophore $\mathbf{4}$ used in this study. TBA is [ $n-$ tetrabutylammonium] $]^{+}$.

In this work, we report investigations into the ion transport behaviour of a series of fluorescent, structurally related benzothioazole substituted SSAs 1-3 in synthetic vesicle and planar bilayers. Our attempts to use existing, literature protocols to study ion transport by these derivatives were complicated by both the intrinsic fluorescence of some structures and the presence of the $\mathrm{TBA}^{+}$counter cation, and hence we developed a novel approach to assaying the $\mathrm{K}^{+}$and $\mathrm{Na}^{+}$transport abilities of this series of molecules. We also report our studies into the deliverability of active transporters, offering insight into the design of synthetic ionophores with improved delivery prospects.

SSAs 1 and $\mathbf{2}$ in addition to anion transporter $\mathbf{4}$ were synthesised in line with previously published methods. ${ }^{30,34}$ SSA 3 was synthesised through the reaction of tetrabutylammonium (TBA) aminomethanesulfonate with 2-aminobenzothiazole and 1,1'-Carbonyldiimidazole (CDI), which gave the desired product as a white solid in a yield of $92 \%$ (see Section S1).

We first sought to investigate whether SSAs 1-3 could mediate cation transport in synthetic phospholipid vesicle systems. Based on literature precedent, we initially explored a $\mathrm{pH}$ gradient assay using the $\mathrm{pH}$ sensitive fluorescent dye HPTS, which is commonly used to assess potential $\mathrm{M}^{+} / \mathrm{H}^{+}$antiport (or $\mathrm{M}^{+} / \mathrm{OH}^{-}$symport) processes. ${ }^{35}$ However, we discovered that the intrinsic fluorescence of SSA 2 precluded the use of this assay. SSA 2 exhibits fluorescence excitation and emission maxima in the same spectral region as the HPTS dye, and thus any changes to the emission of the HPTS were masked by the fluorescence of the prospective carriers. A full discussion of these experiments can be found in Section S2.2. We next attempted a modified $\mathrm{K}^{+} / \mathrm{Na}^{+}$antiport assay, inspired by recent work from Gale, Sessler and Shin, ${ }^{21}$ which uses a potassium selective electrode to monitor $\mathrm{K}^{+}$efflux from phospholipid vesicles. However, we found that the TBA counter cation of SSAs 1-3 caused interference with the potassium electrode (see Section S2.3), with the addition of a solution of TBA nitrate producing a similar response.

Finally, we turned to a chloride co-transport or "dual host" assay, inspired by work reported by Moore et al., which allows the cooperative action of two uniport processes to be assessed (Figure 2, Section S2.4). ${ }^{36}$ We re-designed this assay to avoid the use of fluorescence probes, and instead utilised a chloride ion selective electrode to provide a readout of ion 
transport. POPC vesicles were prepared containing $489 \mathrm{mM} \mathrm{KCl}$, and suspended in $167 \mathrm{mM}$ $\mathrm{Na}_{2} \mathrm{SO}_{4}$, (the ionic strength was maintained at $500 \mathrm{mM}$; all solutions were buffered to $\mathrm{pH} 7.2$ with $5 \mathrm{mM}$ sodium phosphate salts). Upon addition of potential transporters, chloride efflux was monitored using a chloride selective electrode. We reasoned that any observed chloride efflux upon addition of the potential transporters would most likely be the result from an overall $\mathrm{K}^{+} / \mathrm{Cl}^{-}$efflux process, which would avoid the build-up of an unfavourable charge gradient. We tested SSAs 1-3 in isolation, and in combination with previously reported anionophore $4,{ }^{34}$ which is a known anion antiporter but cannot mediate $\mathrm{K}^{+} / \mathrm{Cl}^{-}$symport alone. We thus hoped to investigate whether the SSAs could mediate $\mathrm{K}^{+}$uniport, which would lead to cooperative chloride efflux in combination with anionophore 4. 
(a)

(b)
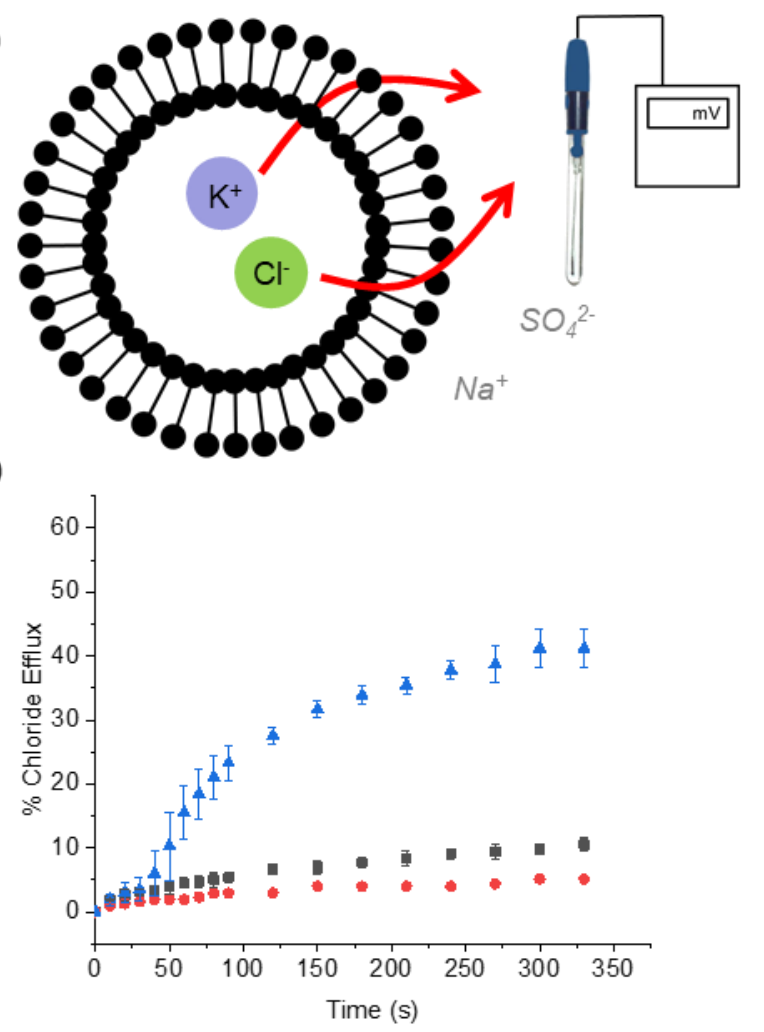

(c)

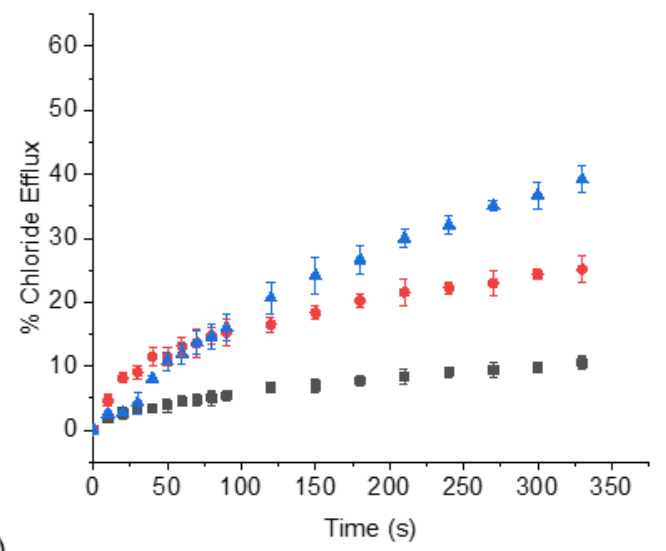

(d)

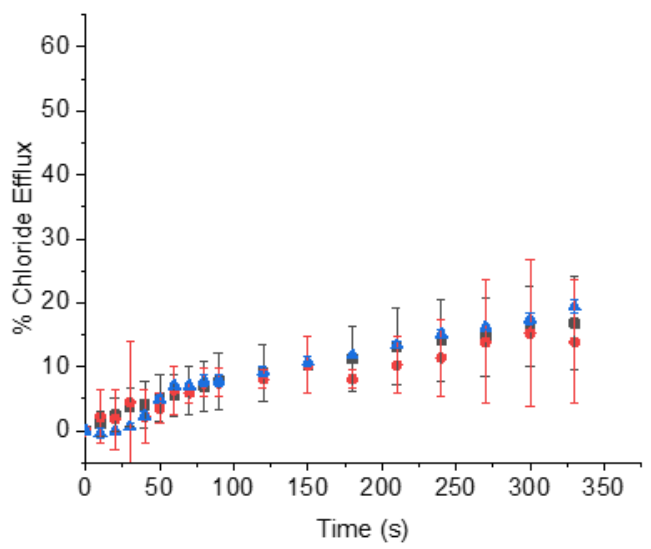

Figure 2 a) Schematic depiction of the $\mathrm{K}^{+} / \mathrm{Cl}$ co-transport assay used in this work. Vesicles containing $\mathrm{KCl}$ were suspended in $\mathrm{Na}_{2} \mathrm{SO}_{4}$. Transporters were added from a DMSO stock solution, and chloride efflux was monitored using a chloride selective electrode. Ions presumed to be innocent in the observed transport are shown in grey italics. (b) results for 
SSA 1 in combination with anionophore 4; (c results for SSA 2 in combination with anionophore 4; (d) results for SSA 3 in combination with anionophore 4. Each data set represents the average of 3 repeat experiments. For all graphs: black squares $=$ anionophore 4 (0.1 mol\% w.r.t. lipid), red circles = SSA (10 mol\% w.r.t. lipid), blue triangles = SSA (10 mol\%) + anionophore 4 (1 mol\%). Error bars represent a standard deviation.

Potential transporters 1-4 were prepared as stock solutions in DMSO. The experiment was initialised by the addition of anionophore 4 ( 1 mol\% w.r.t. lipid), and this was followed by the addition of either DMSO (as a negative control) or SSAs 1-3 (10 mol\% w.r.t. lipid). The activity of the SSAs alone was assessed by adding DMSO followed by the SSA. As expected, the addition of anionophore 4 alone resulted in minimal chloride efflux (Figure $2 b$ d). Likewise, the addition of SSA 1 alone produced minimal chloride efflux (Figure 2b). However, when SSA 1 and anionophore 4 were added in combination, significant chloride efflux was observed (Figure 2b). Importantly, the total chloride efflux was more than the sum of the efflux mediated by $\mathbf{1}$ and $\mathbf{4}$ independently. We thus concluded that $\mathbf{1}$ and $\mathbf{4}$ function together in a cooperative fashion to mediate an overall $\mathrm{K}^{+} / \mathrm{Cl}^{-}$efflux process, and therefore that SSA 1 is able to function as a $\mathrm{K}^{+}$uniporter.

We performed similar experiments using SSAs $\mathbf{2}$ and 3, and found that each of these molecules was able to mediate a small amount of chloride efflux independently of the addition of anionophore 4 (Figures $2 \mathrm{c}$ and $2 \mathrm{~d}$ ). However, the total chloride efflux observed when adding these SSAs in combination with anionophore $\mathbf{4}$ was not significantly larger than the sum of the efflux mediated by the SSA and 4 independently. We hypothesise, that due to the amphiphilic nature of SSAs, that these observations maybe attributed to the surfactant properties of this class compound which result in a small amount vesicle lysis at higher concentrations (see also Section S3).

After observing $\mathrm{K}^{+}$transport by SSA 1 , we next sought to investigate the deliverability of this transporter. Synthetic ionophores are commonly highly lipophilic structures, which can result in poor water solubility and deliverability and therefore limited prospects for medicinal applications. Many synthetic transporters in the literature are tested as stock solutions in organic solvents such as DMSO, acetone or ethanol, and previous work has established that the nature and quantity of the solvent can affect the delivery of the transporter to the lipid bilayer and hence the observed activity. ${ }^{37}$ In contrast, SSAs are charged, amphiphilic salts, and have been reported to dissolve in highly polar, aqueous solvent mixtures including 100 $\% \mathrm{H}_{2} \mathrm{O}^{30}$ and $\mathrm{H}_{2} \mathrm{O} / \mathrm{EtOH}(95: 5) .{ }^{33}$ However, molecular self-association events and the formation of larger aggregates has been observed under these conditions, which might affect the concentration and morphology of the compound to arrive at the surface of the vesicle membrane, and could preclude any active transport activity. ${ }^{30}$ Pleasingly, we found that SSA 1 remained active in our $\mathrm{K}^{+} / \mathrm{Cl}^{-}$co-transport assay when added from a $5 \mathrm{mM}$ stock solution made up in $\mathrm{H}_{2} \mathrm{O}$ / $\mathrm{EtOH}$ (95:5), with some reduction in the observed activity (Figure 3). We performed Hill plot analyses to quantify the transport efficiency of SSA 1 when added from both $\mathrm{DMSO}$ and $\mathrm{H}_{2} \mathrm{O}$ / $\mathrm{EtOH}(95: 5)$ stock solutions, and the results are shown in Table 1. Based on our calculated $\mathrm{EC}_{50}$ values, we established that SSA 1 was around $1.7 \mathrm{x}$ more active when delivered from a DMSO stock solution. We also found that the observed values for $n$, the Hill coefficient, were close to 1 in each case. This could be interpreted as evidence to support a unimolecular transport process; however, we note the potential for Hill plot analyses to be complicated by the formation of stable, aggregate species as described by Matile ${ }^{38}$ since stable supramolecules can be misinterpreted as monomers. Given the extensive data on the dimerization and aggregation of SSAs under a wide range of conditions, ${ }^{29,30}$ we do not believe that these values offer firm proof of a 1:1 SSA: cation transport process. 


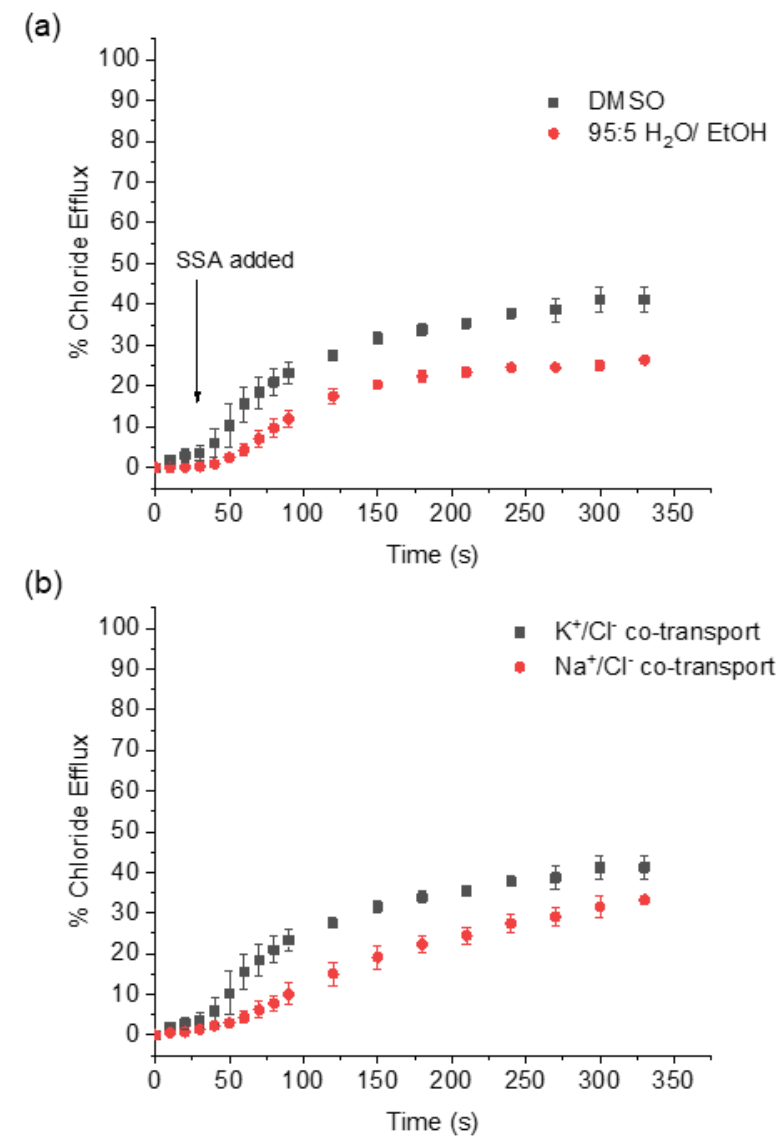

Figure 3 (a) The results from our deliverability study: $\mathrm{K}^{+} / \mathrm{Cl}^{-}$co-transport by SSA 1 (10 mol\%, added from a $5 \mathrm{mM}$ stock solution in either DMSO or $\mathrm{H}_{2} \mathrm{O}$ / $\mathrm{EtOH}(95: 5)$ ) in combination with anionophore 4 (1 mol\%, added from a $10 \mathrm{mM}$ stock solution in DMSO); (b) The results from our cation selectivity study: comparative $\mathrm{K}^{+} / \mathrm{Cl}^{-}$and $\mathrm{Na}^{+} / \mathrm{Cl}^{-}$co-transport by SSA 1 (10 mol\%, added from a $5 \mathrm{mM}$ stock solution in DMSO) in combination with anionophore 4 (1 $\mathrm{mol} \%$, added from a $10 \mathrm{mM}$ stock solution in DMSO).

Table 1 Summary of $M+$ transport results for SSA 1

\begin{tabular}{|l|l|l|}
\hline Stock solution & $\mathbf{E C}_{50}{ }^{\text {a }}$ (mol\% w.r.t. lipid) & $\boldsymbol{n}^{\mathbf{b}}$ \\
\hline $\mathrm{DMSO}, 5 \mathrm{mM}$ & 17.0 & 1.44 \\
\hline $\mathrm{H}_{2} \mathrm{O} / \mathrm{EtOH}(95: 5), 5 \mathrm{mM}$ & 29.1 & 0.95 \\
\hline
\end{tabular}

a Concentration of SSA 1 required to achieve $50 \%$ chloride efflux after 330 s in the presence of $1 \mathrm{~mol} \%$ anionophore 4.

${ }^{b} \mathrm{Hill}$ coefficient for the $\mathrm{M}^{+} / \mathrm{Cl}^{-}$co-transport experiment in the presence of $1 \mathrm{~mol} \%$ of anionophore 4.

We next considered whether SSA 1 could function only as a selective $\mathrm{K}^{+}$uniporter, or whether other biologically relevant cations may also be transported. To investigate this, we adapted our $\mathrm{K}^{+} / \mathrm{Cl}^{-}$co-transport assay to enable us to assay a potential $\mathrm{Na}^{+} / \mathrm{Cl}^{-}$symport process in the presence of anionophore 4. POPC vesicles were prepared containing 489 $\mathrm{mM} \mathrm{NaCl}$, buffered to $\mathrm{pH} 7.2$ with $5 \mathrm{mM}$ sodium phosphate salts. The vesicles were suspended in $167 \mathrm{mM} \mathrm{K}_{2} \mathrm{SO}_{4}$, buffered to $\mathrm{pH}$ 7.2. In short, we reversed the cation gradients from our previously developed assay. The results are shown in Figure $3 \mathrm{~b}$. We found that 
SSA 1 remained active in the $\mathrm{Na}^{+} / \mathrm{Cl}^{-}$co-transport assay with a small reduction in activity, which is consistent with the Hofmeister classification and the greater hydrophilicity of $\mathrm{Na}^{+} \mathrm{vs}$ $\mathrm{K}^{+}$, making it a more challenging ion to transport. Based on this finding, we suggest that SSA 1 could potentially mediate a biologically relevant, $\mathrm{Na}^{+} / \mathrm{K}^{+}$antiport process in the absence of a second co-transporter.

Finally, we sought to assign the ion transport mechanism (carrier or channel) of SSA 1. Based on the small molecular size of this SSA, it cannot feasibly form a unimolecular, membrane-spanning structure; however, given the reported self-assembly capabilities of SSAs, ${ }^{29,}{ }^{30}$ we considered the formation of self-assembled channels a distinct possibility. We performed conductance measurements across DPhPC with $10 \%$ cholesterol planar phospholipid bilayers using a Port-a-Patch miniaturized patch clamp system (see Section S3). This lipid cholesterol mixture was selected as it allowed for the formation of a stable lipid bilayer under these experiment conditions. We found that the addition of SSAs 1-3 and 4 alone did not produce an elevated current recording (Figure 4a), unless at higher concentrations of SSA which resulted in bilayer rupture (Figure $4 \mathrm{~b}$ ). However, under specific conditions, the addition of SSA 1 and anionophore $\mathbf{4}$ together produced an erratic, elevated current flow across the clamped portion of the bilayer (Figure 4c). Control experiments confirmed that these experimental results were not recorded as a result of bilayer rupture. The observed data was not consistent with the controlled, stepwise, open/ close behaviour that is commonly reported for biological and synthetic ion channels. We therefore suggest that SSA 1 operates in combination with anionophore 4 via a mobile carrier mechanism, thus we observe bulk ion transport events.

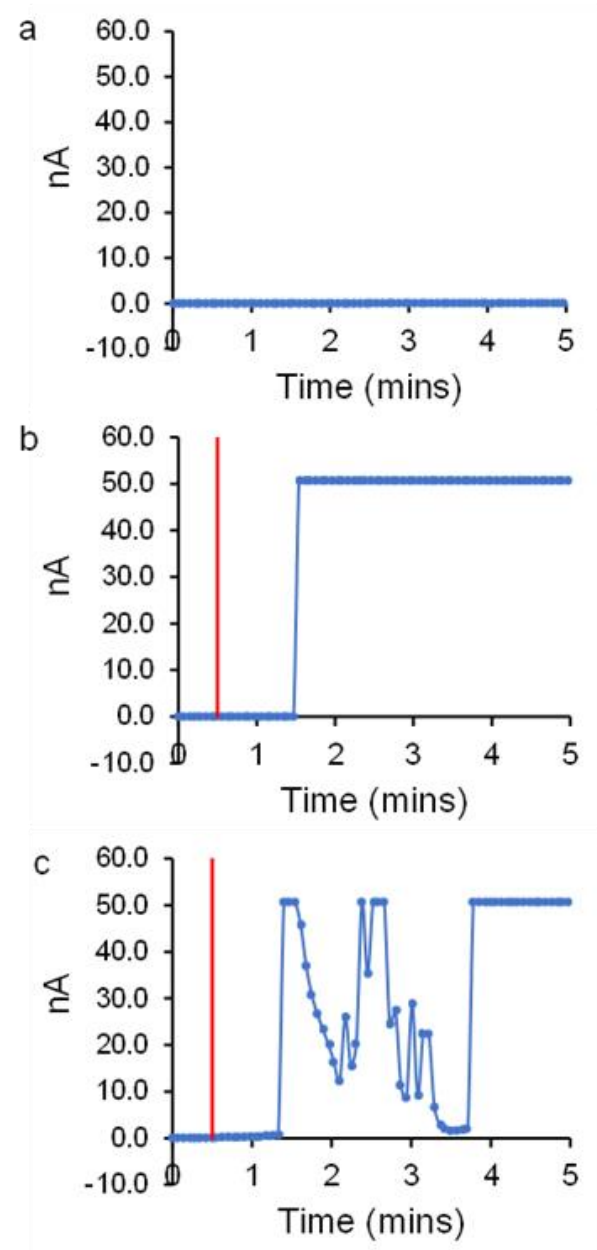


Figure 4 Experimental recording of a DPhPC lipid bilayer at $100 \mathrm{mV}$ after the addition of (a) $4(0.01 \mathrm{mM})$ at 0 seconds b) SSA $1(0.375 \mathrm{mM})$ at 30 seconds and c) $4(0.01 \mathrm{mM})$ at 0 seconds and SSA $1(0.375 \mathrm{mM})$ at 30 seconds. Internal buffer $(489 \mathrm{mM} \mathrm{KCl}$, acetate $5 \mathrm{mM}$, $\mathrm{pH}$ 5.5, ionic strength $500 \mathrm{mM}$ ) and external buffer (167 $\mathrm{mM} \mathrm{Na}_{2} \mathrm{SO}_{4}$, acetate $5 \mathrm{mM}, \mathrm{pH} 5.5$, ionic strength $500 \mathrm{mM}$ ). The red line indicates the addition of SSA 1.

In conclusion, we have reported an investigation of the ion transport activity for a series of supramolecular, self-associating amphiphiles with established antimicrobial and therapeutic enhancement activity. SSA 1 was found to mediate both $\mathrm{K}^{+} / \mathrm{Cl}^{-}$and $\mathrm{Na}^{+} / \mathrm{Cl}^{-}$co-transport in combination with anionophore 4, whereas SSAs $\mathbf{2}$ and $\mathbf{3}$ were not found to mediate selective ion transport and may instead function as membrane disrupting agents. SSA 1 is a novel and relatively hydrophilic transport motif which could be dissolved in and delivered to vesicle bilayers in an aqueous $\mathrm{H}_{2} \mathrm{O}$ / $\mathrm{EtOH}$ (95:5) solvent mixture, demonstrating that charged amphiphiles can have promising deliverability prospects and address major concerns in the development of synthetic ionophores for medicinal applications. We propose that SSA 1 may promote $\mathrm{Na}^{+} / \mathrm{K}^{+}$antiport in the absence of a co-transporter, and that this membrane permeabilisation may be partially responsible for the established biological activity of this molecule. It appears that this is not a generalisable observation that can be applied across this class of SSA molecules, which suggests that different members of this structurally related series may in fact exhibit distinct mechanisms of biological activity.

\section{Funding}

CJEH, YL and HYL gratefully acknowledge financial support from UCL. CJEH and KY also gratefully acknowledge financial support from EPSRC (EP/T025735/1). JH and LW would like to thank the UKRI for JH's Future Leaders Fellowship (MR/T020415/1). JB would like to thank UKHSA and the University of Kent for funding her PhD. KH would also like to thank the University of Kent for funding.

\section{Conflict of interest}

There are no conflicts of interest to declare.

\section{References}

1. B. Aslam, W. Wang, M. I. Arshad, M. Khurshid, S. Muzammil, M. H. Rasool, M. A. Nisar, R. F. Alvi, M. A. Aslam, M. U. Qamar, M. K. F. Salamat and Z. Baloch, Infect. Drug Resist., 2018, 11, 1645-1658.

2. K. A. Brogden, Nature Reviews Microbiology, 2005, 3, 238-250.

3. S. Lin, H. Liu, E. B. Svenningsen, M. Wollesen, K. M. Jacobsen, F. D. Andersen, J. Moyano-Villameriel, C. N. Pedersen, P. Nørby, T. Tørring and T. B. Poulsen, Nat. Chem., 2021, 13, 47-55.

4. N. Akhtar, O. Biswas and D. Manna, Chem. Commun., 2020, 56, 14137-14153.

5. C. R. Elie, G. David and A. R. Schmitzer, J. Med. Chem., 2015, 58, 2358-2366.

6. A. I. Share, K. Patel, C. Nativi, E. J. Cho, O. Francesconi, N. Busschaert, P. A. Gale, S. Roelens and J. L. Sessler, Chem. Commun., 2016, 52, 7560-7563.

7. I. Carreira-Barral, C. Rumbo, M. Mielczarek, D. Alonso-Carrillo, E. Herran, M. Pastor, A. Del Pozo, M. García-Valverde and R. Quesada, Chem. Commun., 2019, 55, 10080-10083.

8. S. Fernandez-Lopez, H.-S. Kim, E. C. Choi, M. Delgado, J. R. Granja, A. Khasanov, K. Kraehenbuehl, G. Long, D. A. Weinberger, K. M. Wilcoxen and M. R. Ghadiri, Nature, 2001, 412, 452-455. 
9. W. M. Leevy, G. M. Donato, R. Ferdani, W. E. Goldman, P. H. Schlesinger and G. W. Gokel, J. Am. Chem. Soc., 2002, 124, 9022-9023.

10. Y. H. Li, S. Zheng, Y. M. Legrand, A. Gilles, A. Van der Lee and M. Barboiu, Angew. Chem. Int. Ed., 2018, 57, 10520-10524.

11. W.-Z. Wang, L.-B. Huang, S.-P. Zheng, E. Moulin, O. Gavat, M. Barboiu and N. Giuseppone, J. Am. Chem. Soc., 2021, 143, 15653-15660.

12. M. Barboiu, Acc. Chem. Res., 2018, 51, 2711-2718.

13. Z. Zhao, B. Tang, X. Yan, X. Wu, Z. Li, P. A. Gale and Y.-B. Jiang, Frontiers of Chemical Science and Engineering, 2021, DOI: 10.1007/s11705-021-2049-7.

14. S. Schneider, E.-D. Licsandru, I. Kocsis, A. Gilles, F. Dumitru, E. Moulin, J. Tan, J.M. Lehn, N. Giuseppone and M. Barboiu, J. Am. Chem. Soc., 2017, 139, 3721-3727.

15. R. Ye, C. Ren, J. Shen, N. Li, F. Chen, A. Roy and H. Zeng, J. Am. Chem. Soc., 2019, 141, 9788-9792.

16. C. Ren, F. Chen, R. Ye, Y. S. Ong, H. Lu, S. S. Lee, J. Y. Ying and H. Zeng, Angew. Chem. Int. Ed., 2019, 58, 8034-8038.

17. C. Ren, J. Shen and H. Zeng, J. Am. Chem. Soc., 2017, 139, 12338-12341.

18. S. Chen, Y. Wang, T. Nie, C. Bao, C. Wang, T. Xu, Q. Lin, D.-H. Qu, X. Gong, Y. Yang, L. Zhu and H. Tian, J. Am. Chem. Soc., 2018, 140, 17992-17998.

19. X.-H. Yu, X.-J. Cai, X.-Q. Hong, K. Y. Tam, K. Zhang and W.-H. Chen, Future Med. Chem., 2019, 11, 1091-1106.

20. J. H. Lee, J. H. Lee, Y. R. Choi, P. Kang, M.-G. Choi and K.-S. Jeong, J. Org. Chem., 2014, 79, 6403-6409.

21. S.-H. Park, I. Hwang, D. A. McNaughton, A. J. Kinross, E. N. W. Howe, Q. He, S. Xiong, M. D. Kilde, V. M. Lynch, P. A. Gale, J. L. Sessler and I. Shin, Chem, 2021, 7, 3325-3339.

22. K. T. Savjani, A. K. Gajjar and J. K. Savjani, ISRN Pharm., 2012, 2012, 10.

23. H. Li, H. Valkenier, L. W. Judd, P. R. Brotherhood, S. Hussain, J. A. Cooper, O. Jurček, H. A. Sparkes, D. N. Sheppard and A. P. Davis, Nat. Chem., 2016, 8, 24-32.

24. J. A. Malla, V. K. Sharma, M. Lahiri and P. Talukdar, Chem. -Eur. J., 2020, 26, 11946-11949.

25. D. A. McNaughton, T. Y. To, B. A. Hawkins, D. E. Hibbs and P. A. Gale, Org. Biomol. Chem., 2021, 19, 9624-9628.

26. J. T. Davis, P. A. Gale and R. Quesada, Chem. Soc. Rev., 2020, 49, 6056-6086.

27. S.-P. Zheng, L.-B. Huang, Z. Sun and M. Barboiu, Angew. Chem. Int. Ed., 2021, 60, 566-597.

28. N. Allen, L. J. White, J. E. Boles, G. T. Williams, D. F. Chu, R. J. Ellaby, H. J.

Shepherd, K. K. L. Ng, L. R. Blackholly, B. Wilson, D. P. Mulvihill and J. R. Hiscock, ChemMedChem, 2020, 15, 2193-2205.

29. L. J. White, S. N. Tyuleva, B. Wilson, H. J. Shepherd, K. K. L. Ng, S. J. Holder, E. R. Clark and J. R. Hiscock, Chem. -Eur. J., 2018, 24, 7761-7773.

30. L. J. White, N. J. Wells, L. R. Blackholly, H. J. Shepherd, B. Wilson, G. P. Bustone, T. J. Runacres and J. R. Hiscock, Chem. Sci., 2017, 8, 7620-7630.

31. J. E. Boles, R. J. Ellaby, H. J. Shepherd and J. R. Hiscock, RSC Adv., 2021, 11, 9550-9556.

32. N. O. Dora, E. Blackburn, J. E. Boles, G. T. Williams, L. J. White, S. E. G. Turner, J. D. Hothersall, T. Askwith, J. A. Doolan, D. P. Mulvihill, M. D. Garrett and J. R. Hiscock, RSC Adv., 2021, 11, 14213-14217.

33. L. J. White, J. E. Boles, N. Allen, L. S. Alesbrook, J. M. Sutton, C. K. Hind, K. L. F. Hilton, L. R. Blackholly, R. J. Ellaby, G. T. Williams, D. P. Mulvihill and J. R. Hiscock, J. Mater. Chem. B, 2020, 8, 4694-4700.

34. S. J. Moore, M. Wenzel, M. E. Light, R. Morley, S. J. Bradberry, P. Gómez-Iglesias, V. Soto-Cerrato, R. Pérez-Tomás and P. A. Gale, Chem. Sci., 2012, 3, 2501-2509.

35. Y.-H. Li, S. Zheng, Y.-M. Legrand, A. Gilles, A. Van der Lee and M. Barboiu, Angew. Chem. Int. Ed., 2018, 57, 10520-10524. 
36. S. J. Moore, M. G. Fisher, M. Yano, C. C. Tong and P. A. Gale, Chem. Commun., 2011, 47, 689-691.

37. C. J. E. Haynes, N. Busschaert, I. L. Kirby, J. Herniman, M. E. Light, N. J. Wells, I. Marques, V. Felix and P. A. Gale, Org. Biomol. Chem., 2014, 12, 62-72.

38. S. Bhosale and S. Matile, Chirality, 2006, 18, 849-856. 